\title{
Minimum of the order parameter fluctuations of seismicity before major earthquakes in Japan
}

\author{
Nicholas V. Sarlis ${ }^{a}$, Efthimios S. Skordas ${ }^{a}$, Panayiotis A. Varotsos ${ }^{a}$, Toshiyasu Nagao ${ }^{b}$, Masashi Kamogawac, \\ Haruo Tanaka ${ }^{d}$, and Seiya Uyeda ${ }^{\mathrm{e}, 1}$
}

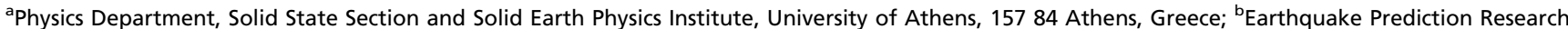
Center, Institute of Ocean Research and Development, Tokai University, Shizuoka 424-8610, Japan; 'Department of Physics, Tokyo Gakugei University, Koganei-shi, Tokyo 184-8501, Japan; ' The Inamori Foundation, Kyoto 600-8411, Japan; and ' Japan Academy, Division 4, Section II, Tokyo 110-0007, Japan
\end{abstract}

Contributed by Seiya Uyeda, July 11, 2013 (sent for review May 18, 2013)

It has been shown that some dynamic features hidden in the time series of complex systems can be uncovered if we analyze them in a time domain called natural time $\chi$. The order parameter of seismicity introduced in this time domain is the variance of $\chi$ weighted for normalized energy of each earthquake. Here, we analyze the Japan seismic catalog in natural time from January 1, 1984 to March 11, 2011, the day of the M9 Tohoku earthquake, by considering a sliding natural time window of fixed length comprised of the number of events that would occur in a few months. We find that the fluctuations of the order parameter of seismicity exhibit distinct minima a few months before all of the shallow earthquakes of magnitude 7.6 or larger that occurred during this 27-y period in the Japanese area. Among the minima, the minimum before the M9 Tohoku earthquake was the deepest. It appears that there are two kinds of minima, namely precursory and nonprecursory, to large earthquakes.

criticality | seismic electric signals

F or a time series comprised of $N$ events, we define the natural time for the occurrence of the $k$ th event by $\chi_{k}=k / N(1)$, which means that we ignore the time intervals between consecutive events, but preserve their order. We also preserve their energy $Q_{k}$. We then study the evolution of the pair $\left(\chi_{k}, p_{k}\right)$, where $p_{k}=$ $Q_{k} / \sum_{n=1}^{N} Q_{n}$ is the normalized energy. We postulated that the approach of a dynamical system to criticality can be identified by the variance $\kappa_{1}$ of natural time $\chi$ weighted for $p_{k}$, namely,

$$
\kappa_{1}=\sum_{k=1}^{N} p_{k} \chi_{k}^{2}-\left(\sum_{k=1}^{N} p_{k} \chi_{k}\right)^{2} \equiv\left\langle\chi^{2}\right\rangle-\langle\chi\rangle^{2} .
$$

Earthquakes (EQs hereafter) exhibit complex correlations in time, space, and magnitude, and the opinion prevails (e.g., ref. 2 and references therein) that the EQs are critical phenomena. In natural time analysis of seismicity, the quantity $\kappa_{1}$ calculated from seismic catalogs serves as an order parameter $(3,4)$. Experiences have shown that the mainshock occurs in a few days to $1 \mathrm{wk}$ after the $\kappa_{1}$ value in the candidate epicentral area approaches 0.070 (5). This was found useful in narrowing the lead time of EQ prediction. However, to trace the time evolution of $\kappa_{1}$ value, one needs to start the analysis of the seismic catalog at some time before the yet-to-occur mainshock. We chose, for the starting time for analysis, the initiation time of seismic electric signal (SES) activity. SESs are low-frequency $(\leq 1 \mathrm{~Hz})$ electric signals that precede EQs (6). The reason for this choice was based on the consideration that SESs are emitted when the focal zone enters the critical stage (7). In the case of the lack of SES data, as in the Tohoku EQ, we cannot adopt this approach. In this study, therefore, we instead examine the fluctuations of $\kappa_{1}$ near criticality, i.e., near the EQ occurrence. To compute the fluctuations, we apply the following procedure.
First, take an excerpt comprised of $W(\geq 100)$ successive EQs from the seismic catalog. We then form its subexcerpts consisting of the $n$th to $(n+5)$ th EQs, $(n=1,2, \ldots, W-5)$ and compute $\kappa_{1}$ for each of them. In so doing, we assign $\chi_{k}=k / 6$ and the normalized energy $p_{k}=Q_{k} / \sum_{n=1}^{6} Q_{n}, k=1,2, \ldots, 6$ to the $k$ th member of the subexcerpt. Note that at least 6 EQs are needed for obtaining reliable $\kappa_{1}(3)$. We iterate the same process for new subexcerpts consisting of 7 members, 8 members, ..., and finally $W$ members Then, we compute the average $\mu\left(\kappa_{1}\right)$ and the SD $\sigma\left(\kappa_{1}\right)$ of the thus-obtained ensemble of $(W-4)(W-5) / 2 \kappa_{1}$ values. The variability $(4,8)$ of $\kappa_{1}$ for this excerpt $W(\geq 100)$ is defined to be $\beta \equiv \sigma\left(\kappa_{1}\right) / \mu\left(\kappa_{1}\right)$ and is assigned to the $(W+1)$ th EQ, the target EQ.

The time evolution of the $\beta$-value can be pursued by sliding the excerpt through the EQ catalog. Through the same process as explained above, the $\beta$-values to be assigned to the $(W+2)$ th, $(W+3)$ th, EQs in the catalog will be obtained.

\section{Data Analyzed}

For our analysis, we used the Japan Meteorological Agency (JMA) seismic catalog and considered all of the EQs in the period from 1984 to the time of the M9 Tohoku EQ, within the area $25^{\circ}-46^{\circ} \mathrm{N}, 125^{\circ}-148^{\circ} \mathrm{E}$, which covers the whole Japanese region (Fig. 1). The energy of EQs was obtained from $\mathrm{M}_{\mathrm{JMA}}$ after converting (9) to the moment magnitude $M_{w}$ defined by Kanamori (10). Setting a threshold $\mathrm{M}_{\mathrm{JMA}}=3.5$ to assure the data completeness, we are left with 47,204 EQs in the concerned period of about 326 mo. Thus, we have on the average $\sim 10^{2} \mathrm{EQs}$ per month. We chose the values $W=200,300$, and 400, which would cover a period of a few months before each target EQ. This choice of a few months is based on the experience that the lead time of SES activities is of this order both in Japan (11) and Greece $(5,7,12)$.

\section{Minimum of the Variability $\beta$ Before the M9 Tohoku EQ}

Fig. $2 A$ depicts about 47,200 $\beta$-values calculated for $W=300$ versus the target EQ number from 1984 to the day of the Tohoku EQ, March 11, 2011. EQs with $\mathrm{M}_{\mathrm{JMA}} \geq 6.9$ (M $_{\mathrm{JMA}}$ in the right scale) are shown by blue asterisks. One can see that $\beta$-values fluctuate up and down so violently that it is hard to identify their correlations with EQs. However, one can notice that $\beta$ shows a deep minimum value just before the Tohoku EQ (rightmost side of Fig. $2 A$ ). This observation prompted us to investigate more about this $\beta$ minimum. Fig. $2 B$ is an expanded version, in the conventional time, of the concerned part of Fig. $2 A$ (the last 10 -mo period shown in yellow). The red, blue, and green curves show what happened to $\beta$ for $W=200,300$, and 400 . For brevity,

Author contributions: N.V.S. and P.A.V. designed research; N.V.S., E.S.S., P.A.V., T.N., M.K. H.T., and S.U. performed research; N.V.S. and E.S.S. analyzed data; and N.V.S., E.S.S., P.A.V. T.N., M.K., and S.U. wrote the paper.

The authors declare no conflict of interest.

${ }^{1}$ To whom correspondence should be addressed. E-mail: suyeda@st.rim.or.jp. 


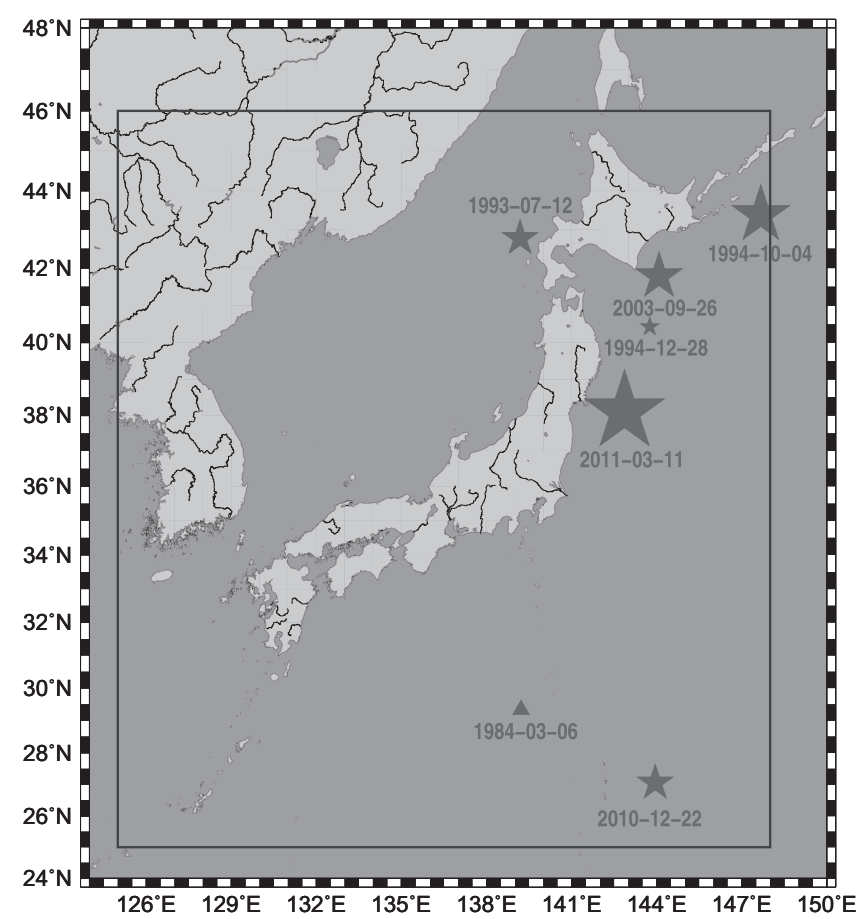

Fig. 1. Epicenters (stars) of all major EQs with magnitude 7.6 or larger within the area $N_{25}^{46} E_{125}^{148}$ since January 1, 1984 until the M9 Tohoku EQ (Table 1). The deep EQ on March 6, 1984 is depicted by a triangle.

we use hereafter the symbols $\beta_{W}$ and $\beta_{W \text {,min }}$ as needed. Putting the details aside, we observe that after around September 1, 2010 a decrease of $\beta_{W}$ became evident and $\beta_{W}$ went down to a minimum $\left(\beta_{200, \text { min }} \sim 0.157, \beta_{300 \text {, min }} \sim 0.160\right.$, and $\left.\beta_{400, \text { min }} \sim 0.150\right)$ in early January 2011, about 2 mo before the mainshock. (The abrupt increase of $\beta$ around December 22, 2010 was due to the M7.8 EQ on this date, e.g., ref. 3.)

Results of the computation on this minimum of $\beta$ are summarized as follows (Fig. $2 A$ and $B$, and Table 1):

$i$ ) Minimum of $\beta$ with this depth was not observed at any other time during the whole period.

ii) $\beta_{300, \min } / \beta_{200, \min }=0.160 / 0.157=1.02$, i.e., nearly unity.

iii) The dates of $\beta_{W \text {,min }}$ for $W=200,300$, and 400 are January 5, January 5, and January 10, 2011, respectively, i.e., the dates of $\beta_{W \text {,min }}$ were almost the same.

iv) The appearance of this minimum is less clear for greater $W$ that would correspond to time intervals longer than a few months. It is almost invisible for $W=2,000$ and 3,000. (Fig. $2 C$ ). The same applies to all other $\beta_{W \text { min }}$ as seen in Fig. $2 A$ and $C$. In what follows, for the sake of brevity we shall restrict ourselves to the cases of $W=200$ and $W=300$.
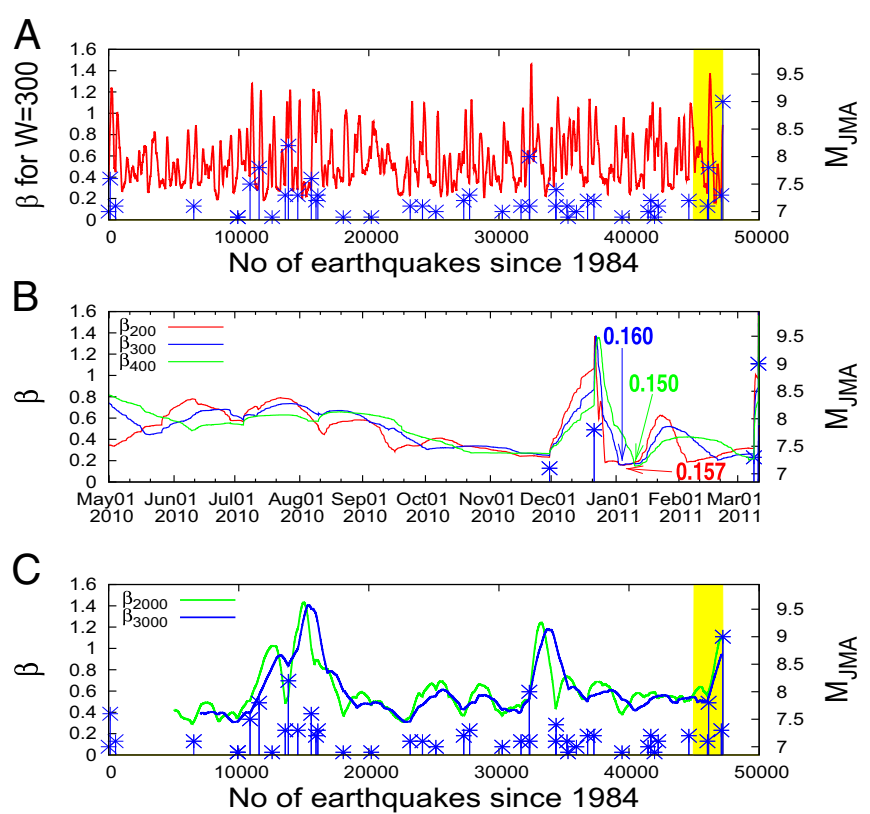

Fig. 2. Variability $\beta$ of $\kappa_{1}$ (left scale) along with all $M_{J M A} \geq 6.9 E Q s$ (in blue, $M_{J M A}$ in the right scale). ( $A$ ) Versus EQ number when a natural time window of length $W=300$ events is sliding through the JMA catalog since 1984 until just before the M9 Tohoku EQ. $(B)$ Versus the conventional time during the last 10-mo period (shown by yellow in $A$ ). Red for $W=200$, blue for $W=300$, and green for $W=400$. Every tick is $10 \mathrm{~d}$ in the horizontal scale. (C) Variability $\beta$ for $W=2,000$ (green) and $W=3,000$ (blue).

\section{Minima of the Variability $\boldsymbol{\beta}$ Before Other Major EQs in Japan}

During the 27-y study period, we had six shallow EQs with $\mathrm{M}_{\text {JMA }}$ 7.6 or larger (Fig. 1 and Table 1). They are

EQa 1993-07-12: 1993 Southwest-Off Hokkaido EQ $\left(\mathrm{M}_{\mathrm{JMA}}=7.8\right)$

EQb 1994-10-04: 1994 East-Off Hokkaido EQ ( JMA $_{\text {JMA }}$ 8.2)

EQc 1994-12-28: 1994 Far-Off Sanriku EQ (M JMA $_{\text {= }}$ 7.6)

EQd 2003-09-26: 2003 Off Tokachi EQ ( JMA $_{\text {JMA }}$ 8.0)

EQe 2010-12-22: 2010 Near Chichi-jima EQ ( MMA $\left._{\text {JMA }} 7.8\right)$

EQf 2011-03-11: 2011 Tohoku EQ (Mw = 9.0)

In the following, we examine if minimum of $\beta$ exists before these EQs also. Fig. $3 A-C$ are the expanded versions of Fig. $2 A$ in the conventional time in three 10 -y periods. EQs are marked by a-f. Because these figures are still too small, we expanded the time axis for each EQ as shown in Fig. $4 A-E$, just as we did for in Fig. $2 B$ for the Tohoku EQ. We can see minima of $\beta$ within 1-3 mo before all of the six mainshocks. In Table 1, these minima are listed along with the time-correlated EQs. As seen in this table, the values of the $\beta_{300, \min } / \beta_{200, \text { min }}$ ratio and $\Delta \mathrm{t}_{200}$ of minima of $\beta$

Table 1. All shallow EQs with magnitude 7.6 or larger since January 1, 1984 until M9 Tohoku EQ within the area $N_{25}^{46} E_{125}^{148}$

\begin{tabular}{|c|c|c|c|c|c|c|c|c|c|}
\hline Label & EQ date & EQ name & Lat., ${ }^{\circ} \mathrm{N}$ & Long., ${ }^{\circ} \mathrm{E}$ & M & $\beta_{200, \text { min }}$ & $\beta_{300, \min }$ & $\beta_{300, \text { min }} / \beta_{200, \text { min }}$ & $\Delta t_{200}$ \\
\hline a & 1993-07-12 & Southwest-Off Hokkaido EQ & 42.78 & 139.18 & 7.8 & $0.293(1993-05-23)$ & $0.278(1993-06-07)$ & 0.95 & 2 \\
\hline b & 1994-10-04 & East-Off Hokkaido EQ & 43.38 & 147.67 & 8.2 & $0.295(1994-06-30)$ & $0.319(1994-07-22)$ & 1.08 & 3 \\
\hline c & $1994-12-28$ & Far-Off Sanriku EQ & 40.43 & 143.75 & 7.6 & $0.196(1994-10-15)$ & 0.197 (1994-10-19) & 1.01 & $2-3$ \\
\hline e & $2010-12-22$ & Near Chichi-jima EQ & 27.05 & 143.94 & 7.8 & $0.232(2010-11-30)$ & $0.248(2010-11-30)$ & 1.07 & 1 \\
\hline$f$ & 2011-03-11 & Tohoku EQ & 38.10 & 142.86 & 9.0 & $0.157(2011-01-05)$ & $0.160(2011-01-05)$ & 1.02 & 2 \\
\hline
\end{tabular}

The symbols $\beta_{W, \min }$ are the minima of the $\kappa_{1}$ variability that preceded these EQs along with their dates. $\Delta \mathrm{t}_{200}$ is the difference in months between the dates of $\beta_{200 \text {, min }}$ and EQ. Lat., latitude; Long., longitude. 

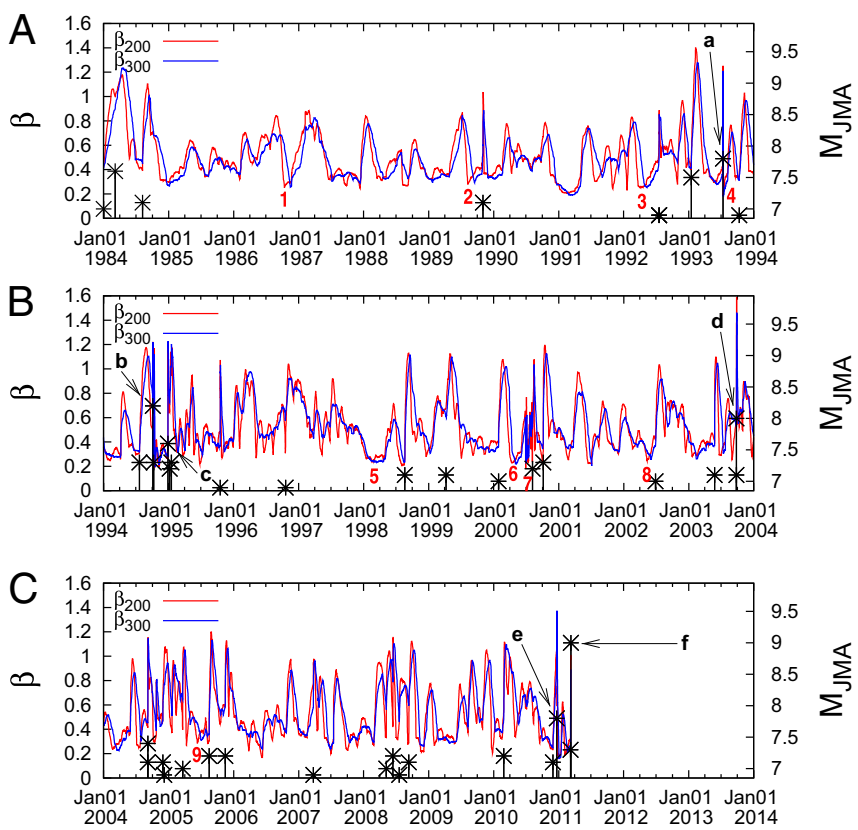

Fig. 3. Variability $\beta$ versus the conventional time given in three consecutive 10 -y periods in $A, B$, and $C$, respectively. No data are plotted in $C$ after $\mathrm{M} 9$ Tohoku EQ. Every tick is $3 \mathrm{mo}$ in the horizontal scale.

before all of these EQs were very similar to those observed before the Tohoku EQ, i.e., they were in the narrow range 0.95 1.08. From these, it was considered that these minima may be precursory to the time-correlated EQs.

During the 27-y period, beyond the above-mentioned minima of $\beta$ before the six $\mathrm{M}_{\mathrm{JMA}} \geq 7.6 \mathrm{EQs}$, there were many more minima as seen in Fig. 3. We therefore examined if they were also followed by EQs. For this purpose, we chose minima deeper than the shallowest one of the six $\beta_{200 \text {,min }}$ in Table 1, which happened before $\mathbf{E Q b}$, the 1994 East-Off Hokkaido EQ $\left(\mathrm{M}_{\mathrm{JMA}}=\right.$

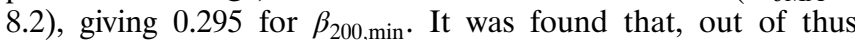
chosen 31 minima, nine (numbered 1-9 in Fig. 3) also exhibited $\beta_{300, \text { min }} / \beta_{200 \text {,min }}$ ratio (Fig. 5, Table 2) similar to those in Table 1, i.e., in the range $0.95-1.08$. These nine minima were followed by $\mathrm{M}_{\text {JMA }} \geq 6.4$ EQs within 3 mo (Fig. 5 and $\Delta t_{200}$ in Table 2). Naturally, such correspondences are less certain because of greater number of EQs. In fact, during the 27-y period, there were $139 \mathrm{M}_{\text {JMA }} \geq 6.4$ EQs. The cases mentioned here, however, may have some reason to be reliable as explained below (Table 2). For example, the EQ that apparently followed $\beta_{W \text {,min }}$ No. 6 in April 2000 was the largest EQ of the volcano-seismic activity in the Izu Island area (11), the largest EQ swarm ever recorded
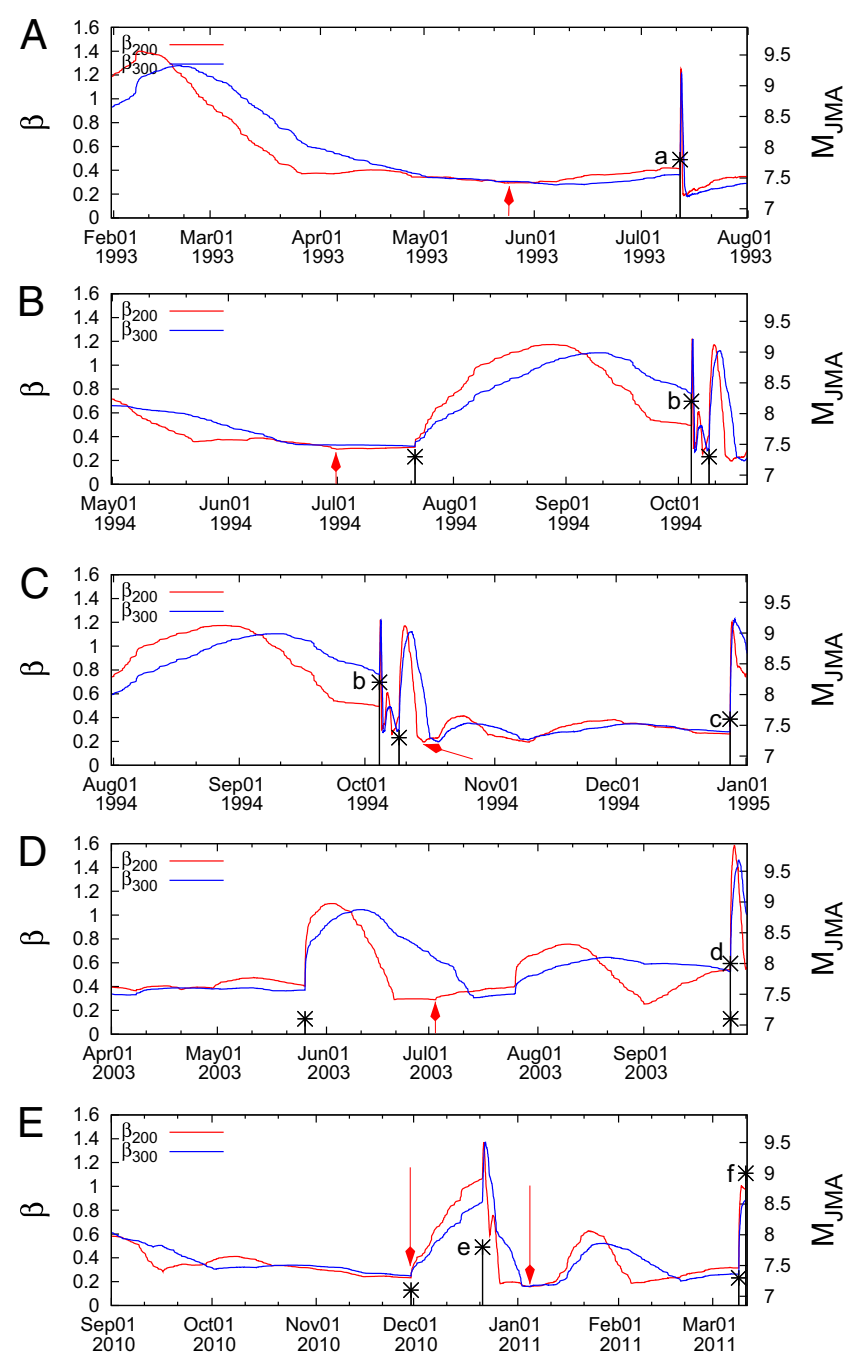

Fig. 4. Excerpts of Fig. 3 plotted in expanded time scale. Every tick is $10 \mathrm{~d}$ in the horizontal scale. Red arrows show $\beta_{200 \text {,min }}$ along with EQs (in black a-f $\mathrm{M}_{\mathrm{JMA}}$ in the right scale). The figures show what happened for the variability $\beta$ before the occurrence of $(A)$ EQa, $(B)$ EQb, $(C)$ EQb and EQc, $(D)$ EQd, and (E) EQe and EQf (the M9 Tohoku EQ).

in Japan. For a second example, $\beta_{W \text {,min }}$ No. 7 in July 2000 was followed by $\mathrm{M}_{\mathrm{JMA}}$ 7.3 Western Tottori EQ. The period after this EQ in the year 2000 was completely free from shallow EQ greater than $\mathrm{M}_{\mathrm{JMA}}$ 6. Other examples are the minima Nos. 9, 2, 8, 3, and 4 which were followed by EQs of $\mathrm{M}_{\mathrm{JMA}}$ 7.2, 7.1, 7.0, 6.9,

Table 2. Nine $\boldsymbol{\beta}_{W, \min }$ of the $\kappa_{1}$ variability not included in Table 1 but chosen by the procedure described in the text

\begin{tabular}{lcccccccc} 
No. & $\beta_{200, \min }$ & $\beta_{300, \min }$ & $\beta_{300, \min } / \beta_{200, \min }$ & $\mathrm{EQ}$ date & Lat., ${ }^{\circ} \mathrm{N}$ & Long., ${ }^{\circ} \mathrm{E}$ & $\mathrm{M}$ & $\Delta \mathrm{t}_{200,} \mathrm{mo}$ \\
\hline 1 & $0.254(1986-10-13)$ & $0.257(1986-11-15)$ & 1.01 & $1987-01-14$ & 42.45 & 142.93 & 6.6 & 3 \\
2 & $0.278(1989-08-08)$ & $0.292(1989-09-15)$ & 1.05 & $1989-11-02$ & 39.86 & 143.05 & 7.1 & 3 \\
3 & $0.250(1992-04-05)$ & $0.253(1992-05-10)$ & 1.01 & $1992-07-18$ & 39.37 & 143.67 & 6.9 & 3 \\
4 & $0.188(1993-07-13)$ & $0.182(1993-07-15)$ & 0.97 & $1993-10-12$ & 32.03 & 138.24 & 6.9 & 3 \\
5 & $0.237(1998-02-17)$ & $0.233(1998-03-12)$ & 0.98 & $1998-05-31$ & 39.03 & 143.85 & 6.4 & 3 \\
6 & $0.229(2000-04-12)$ & $0.219(2000-05-06)$ & 0.96 & $2000-07-01$ & 34.19 & 139.19 & 6.5 & 3 \\
7 & $0.243(2000-07-09)$ & $0.258(2000-07-09)$ & 1.06 & $2000-10-06$ & 35.27 & 133.35 & 7.3 & 3 \\
8 & $0.244(2002-05-12)$ & $0.252(2002-06-03)$ & 1.03 & $2002-06-29$ & 43.50 & 131.39 & 7.0 & 2 \\
9 & $0.286(2005-06-11)$ & $0.309(2005-07-01)$ & 1.08 & $2005-08-16$ & 38.15 & 142.28 & 7.2 & 2 \\
\hline
\end{tabular}

The $\beta_{W \text {,min }}$ exhibit $\beta_{200 \text {,min }}$ deeper than 0.295 , which corresponds to the shallowest $\beta_{200, \min }$ in Table 1 . EQs are time-correlated to the $\beta_{200 \text {,min }}$. 

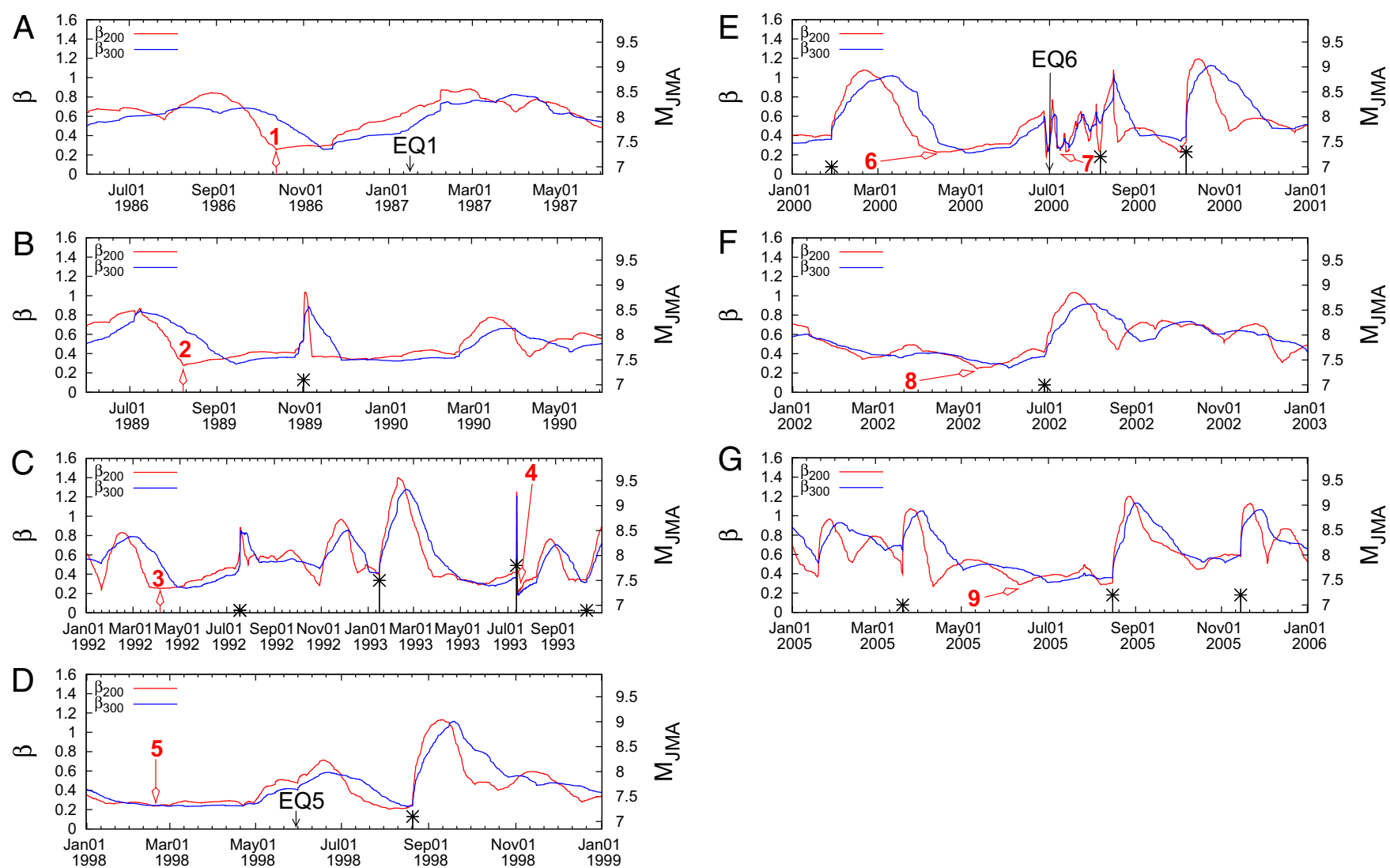

Fig. 5. Excerpts of Fig. 3 but corresponding to each of the nine cases of $\beta_{200, \text { min }}$ given in Table $2:(A) 1,(B) 2,(C) 3$ and 4, $(D) 5,(E) 6$ and $7,(F) 8$, and $(G) 9$. Every tick is 10 $\mathrm{d}$ in the horizontal scale. Numbers 1-9 correspond to $\beta_{200, \text { min }}$ in Fig. 3 and Table 2. EQs time-correlated to minima 1, 5, and 6 are shown with the vertical black arrows.

and 6.9. For brevity, each case is not described here, but it was inferred that these $\beta_{W \text {,min }}$ might have also been precursory to sizable EQs. In fact, there were only $43 \mathrm{M}_{\mathrm{JMA}} \geq 6.9$ EQs during the $27-\mathrm{y}$ period. Likewise, the $\beta_{W \text {,min }}$ Nos. 1 and 5 seemed followed by EQs of $\mathrm{M}_{\mathrm{JMA}} 6.6$ and $\mathrm{M}_{\mathrm{JMA}} 6.4$, respectively, although their correlations are even less certain. After handling these, we are still left with 22 minima unnumbered or unmarked in Fig. 3.

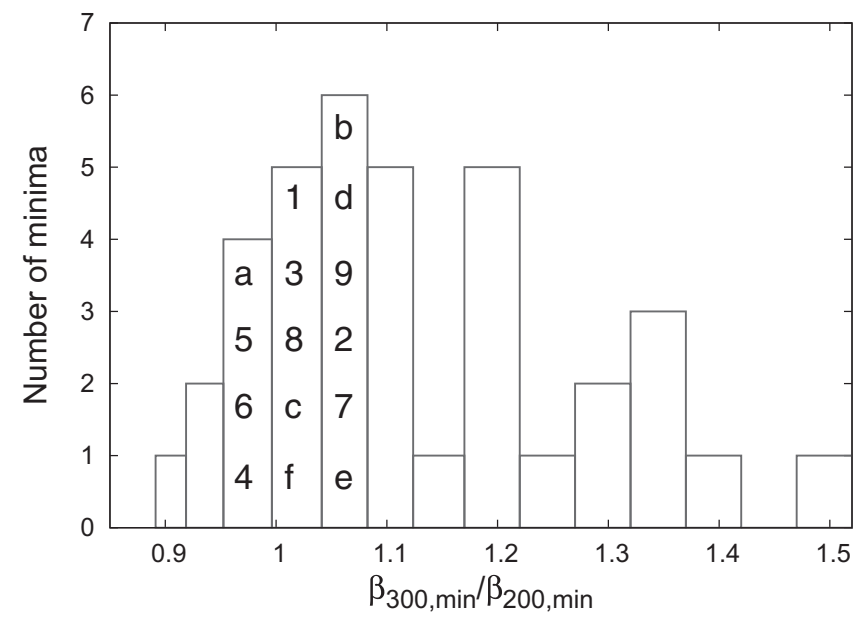

Fig. 6. Histogram of the $\beta_{300, \min } / \beta_{200, \text { min }}$ ratio for the 37 minima in Fig. 3 which are deeper than the shallowest $\beta_{200 \text { min }}$ of Table 1 . The minima marked a-f or numbered 1-9 in Fig. 3 are placed vertically in the corresponding column according to their $\beta_{200 \text { min }}$ values.
We have checked the $\beta_{300, \min } / \beta_{200 \text {,min }}$ ratio of each of them. For example, $\beta_{W \text {,min }}\left(\beta_{200, \text { min }}=0.213\right.$ and $\left.\beta_{300, \text { min }}=0.259\right)$ observed on December 4, 2008 (Fig. $3 C$ ) exhibited a ratio $\beta_{300, \text { min }} / \beta_{200 \text {,min }}$ $(=1.22)$, which lies outside the range $0.95-1.08$. Fig. 6 is the histogram of the $\beta_{300, \min } / \beta_{200 \text {,min }}$ ratio for all of the 37 minima examined so far, consisting of the 6 in Table 1 marked a-f, 9 in Table 2 marked $1-9$, and the 22 additionally chosen minima. From this figure, interestingly, none of the additional 22 minima exhibits the ratio within the range 0.95-1.08.

\section{Summary and Conclusions}

Analyzing in natural time the seismicity of Japan from January 1, 1984 to March 112011 (the time of M9 Tohoku EQ occurrence), using sliding natural time window of lengths $W$ consisting of the number of events that would occur in a few months, the following results were obtained:

Almost 2 mo before the M9 Tohoku EQ, a minimum in the variability $\beta$ of the order parameter of seismicity $\kappa_{1}$ is observed which is the deepest in the whole study period. Distinct minima of $\beta$, but of shallower depth, were found also one month to a few months before the occurrence of all other Japanese major EQs $\left(\mathrm{M}_{\mathrm{JMA}} \geq 7.6\right.$, depth $\left.<400 \mathrm{~km}\right)$ during 1984-2011. With less certitude, nine other minima of $\beta$ may have also been precursory to large EQs. The minima of $\beta$ which seem to be precursory to sizable EQ commonly show the $\beta_{300 \text { min }} / \beta_{200 \text { min }}$ ratio close to unity in the range of $0.95-1.08$, whereas the other minima show the ratio outside this range. Thus, the phenomenon of minimum in the $\beta$-value may play some role as a precursor in the EQ prediction in the future.

The approximate coincidence of the lead time of minima of $\beta$ with that of the SES activities may help in understanding the physics of both phenomena. 
1. Varotsos P, Sarlis NV, Skordas ES, Uyeda S, Kamogawa M (2011) Natural time analysis of critical phenomena. Proc Natl Acad Sci USA 108(28):11361-11364.

2. Holliday JR, et al. (2006) Space-time clustering and correlations of major earthquakes. Phys Rev Lett 97(23):238501.

3. Varotsos PA, Sarlis NV, Tanaka HK, Skordas ES (2005) Similarity of fluctuations in correlated systems: The case of seismicity. Phys Rev E Stat Nonlin Soft Matter Phys 72(4 Pt 1):041103.

4. Varotsos PA, Sarlis NV, Skordas ES (2011) Natural Time Analysis: The New View of Time. Precursory Seismic Electric Signals, Earthquakes and other Complex Time-Series (Springer, Berlin), $476 \mathrm{pp}$

5. Sarlis NV, Skordas ES, Lazaridou MS, Varotsos PA (2008) Investigation of seismicity after the initiation of a Seismic Electric Signal activity until the mainshock. Proc. Japan Acad. Ser. B 84(8):331-343.
6. Varotsos P, Alexopoulos K (1984) Physical properties of the variations of the electric field of the earth preceding earthquakes, I. Tectonophysics 110(1-2):73-98.

7. Varotsos P, Alexopoulos K, Lazaridou M (1993) Latest aspects of earthquake prediction in Greece based on Seismic Electric Signals, II. Tectonophysics 224(1-3):1-37.

8. Sarlis NV, Skordas ES, Varotsos PA (2010) Order parameter fluctuations of seismicity in natural time before and after mainshocks. EPL 91(5):59001.

9. Tanaka H, Varotsos P, Sarlis N, Skordas E (2004) A plausible universal behavior of earthquakes in the natural time-domain. Proc Japan Acad, Ser B 80(6):283-289.

10. Kanamori H (1978) Quantification of earthquakes. Nature 271(5644):411-414.

11. Uyeda S, Kamogawa M, Tanaka $H$ (2009) Analysis of electrical activity and seismicity in the natural time domain for the volcanic-seismic swarm activity in 2000 in the Izu Island region, Japan. J Geophys Res 114(B2):B02310.

12. Varotsos P, Lazaridou M (1991) Latest aspects of earthquake prediction in Greece based on Seismic Electric Signals. Tectonophysics 188(3-4):321-347. 